Mon. Not. R. Astron. Soc. 000, 目 (1998) Printed 18 November $2018 \quad$ (MN LATEX style file v1.4)

\title{
High resolution optical and infrared spectroscopic observations of Cir X-1
}

\author{
Helen M. Johnston, ${ }^{1 \star}$ Robert Fender, ${ }^{2}$ and Kinwah $\mathrm{Wu}^{3}$ \\ 1 Anglo-Australian Observatory, P.O. Box 296, Epping NSW 1710, Australia \\ 2 Astronomical Institute 'Anton Pannekoek', University of Amsterdam, Kruislaan 403, 1098 SJ Amsterdam, The Netherlands \\ 3 Research Centre for Theoretical Astrophysics, School of Physics, University of Sydney, NSW 2006, Australia
}

\begin{abstract}
We present new optical and infrared (IR) observations of Cir X-1 taken near apastron. Both sets of spectra show asymmetric emission lines. Archival optical observations show that an asymmetric $\mathrm{H} \alpha$ emission line has been in evidence for the past twenty years, although the shape of the line has changed significantly. We present an eccentric $(e \sim 0.7-0.9)$ low mass binary model, where the system consists of a neutron star orbiting around a (sub-)giant companion star of 3-5 $\mathrm{M}_{\odot}$. We suggest that the broad components of the emission lines arise in a high-velocity, optically thick flow near the neutron star; while the narrow components of the optical and the IR lines arise near the companion star and a heated ejecta-shell surrounding the binary respectively. In this model, the velocity of the narrow component reflects the space velocity of the binary; the implied radial velocity $\left(+430 \mathrm{~km} \mathrm{~s}^{-1}\right.$ after correcting for Galactic rotation) is the highest velocity known for an X-ray binary.
\end{abstract}

Key words: binaries: spectroscopic - stars: individual: Cir X-1 - stars: X-rays

\section{INTRODUCTION}

Cir X-1 is one of the most puzzling X-ray binaries known. Like the peculiar systems SS 433 and Cyg X-3, it cannot easily be classified into any of the major categories of X-ray binaries. Indeed, there is even doubt as to whether it is a high-mass (HMXB) or low-mass X-ray binary (LMXB).

Since its discovery in early 1970s, Cir X-1 has been studied intensively at X-ray wavelengths. The X-ray properties of Cir X-1 were found to differ dramatically each time it was observed (see for example khbs76,ten88,tkm+89,sblm96 ) . Periodic modulation of the X-ray flux was found at a period of $16.6 \mathrm{~d}$ (khbs76 ) . A radio counterpart was found (cpc75 ), and was found to flare at the same period as the X-ray modulation $(\mathrm{hjm}+78)$. These flares were initially detected at peak flux levels of $>1 \mathrm{Jy}$; since the 1970s, the flux of the source has decreased dramatically, and it has only occasionally been detected above $50 \mathrm{mJy}(\mathrm{snp}+91)$.

This radio source is located $25^{\prime}$ from the centre of the supernova remnant G321.9-0.3, and is apparently connected to the remnant by a radio nebula $(\mathrm{hkl}+86) . \mathrm{schn} 93(\mathrm{~h})$ ave imaged arcmin-scale collimated structures within the surrounding nebula, suggesting an outflow from the X-ray binary. fst $+98(\mathrm{~h})$ ave imaged an arcsec-scale asymmetric jet aligned with these larger structures, raising the possibility

\footnotetext{
^ E-mail: hmj@aaoepp.aao.gov.au
}

that the outflow from the system is relativistic. Recently, cb98 (h) ave revised the estimated distance to G321.9-0.3 (and hence to Cir X-1, assuming they are associated) to $5.5 \mathrm{kpc}$, which is substantially smaller than the original suggested distance to Cir X-1 of $10 \mathrm{kpc}$ (gm77) .

The discovery of Type I X-ray bursts (tfs86b) suggests that the compact object is probably a weakly-magnetised neutron star. The close association of Cir X-1 with the supernova remnant suggests that the system may be a young $\left(<10^{5}\right.$ y old $)$ runaway system from a supernova explosion (schn93).

The optical counterpart to Cir X-1 was identified as a highly-reddened star with strong $\mathrm{H} \alpha$ emission (wmw +77 ) . This object was later shown to consist of three stars within a radius of $1^{\prime \prime} .5$, the southernmost of which is the true counterpart (mon92,dsh93).

The long orbital period and periodic X-ray activity suggested a high-mass system in an eccentric orbit $(m j h+80)$; however, the variability of the optical emission, the faintness of the optical counterpart, and several of its X-ray characteristics suggest that the companion is a low-mass star. The lack of spectroscopic studies in the optical band means that most of the fundamental orbital parameters of Cir X-1 have not been determined. Moreover, Cir X-1 shows very different properties from time to time. This make it difficult to construct a coherent picture for the system from observations of different wavelengths at different epochs. Here we present new spectroscopic observations of Cir X-1, and use

(C) $1998 \mathrm{RAS}$ 


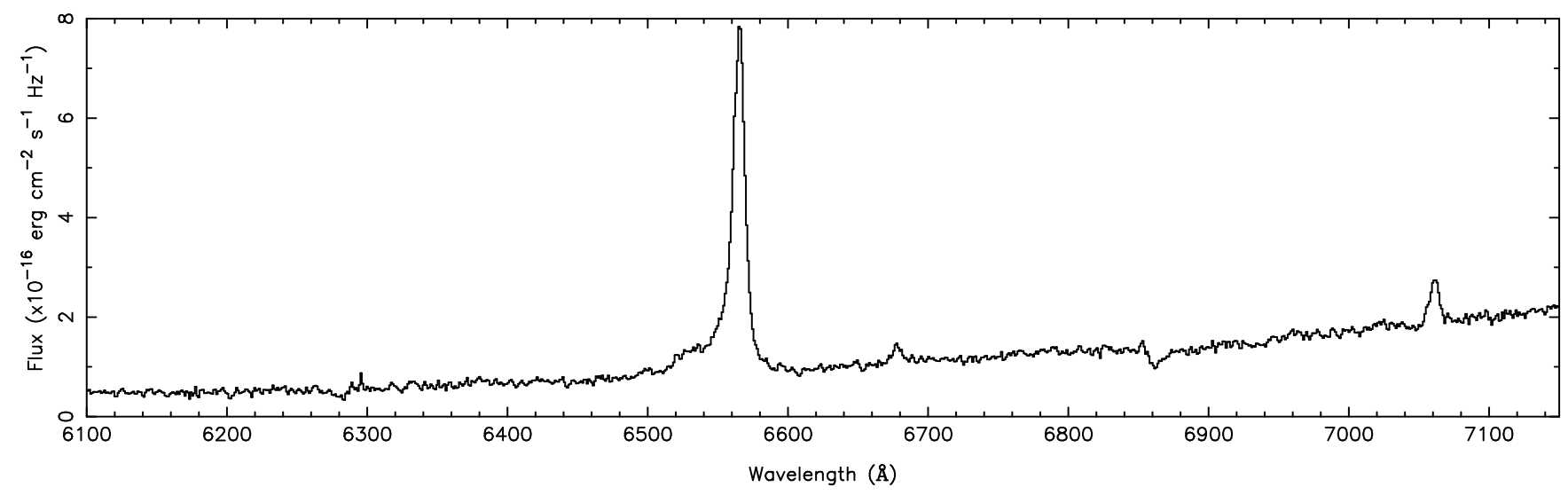

Figure 1. Spectrum of Cir X-1, taken near apastron on 1997 June 4: the spectrum shown is the sum of five $1800 \mathrm{~s}$ exposures. Emission lines of lines of $\mathrm{H} \alpha \lambda 6563$, He I $\lambda 6678$, and He I $\lambda 7065$ can be seen; the dip at $\lambda 6870$ is due to imperfect removal of the terrestrial atmospheric B-band.

these, together with analysis of archival observations of the system, to suggest a more coherent model for the system.

\section{OBSERVATIONS AND DATA REDUCTION}

\subsection{New optical observations}

Cir X-1 was observed on 1997 June 4 using the 3.9 m AngloAustralian Telescope (AAT). The mean orbital phase of the observation was 0.51 , calculated according the ephemeris of snp+91 (.) The RGO Spectrograph was used in combination with the TEK $1 \mathrm{k}$ CCD in the $82 \mathrm{~cm}$ camera and a grating of 270 grooves $\mathrm{mm}^{-1}$ in first order, resulting in a dispersion of $\sim 1.08 \AA$ pixel $^{-1}$ over a wavelength range $6060-7165 \AA$. The spatial scale was 0 .' 25 ; the spectral resolution, measured from the arc lines, was $5.4 \AA$.

A 1.' 5 -wide slit was used, oriented north-south so both Cir X-1 and star 2 of mon92 (w) ere in the slit. The atmospheric seeing was about $1^{\prime \prime}$. Five $1800 \mathrm{~s}$ integrations were taken, interspersed with $\mathrm{CuAr}$ arc-lamp exposures, before cloud prevented the acquisition of any more data.

The bias and pixel-to-pixel gain variations were removed from each exposure using standard procedures in IRAF. Cosmic rays were removed using the method of cro95 (t) o compare adjacent frames. Because of the presence of the nearby confusing star (Moneti's star 2), special care needed to be taken to measure the flux from our object. At every position along the dispersion direction, we fit two gaussian profiles, with fixed widths (FWHM=5.6 pixels) and separation (6 pixels), to the sky-subtracted frames. The amplitude of these gaussians was used as the estimate of the flux from Cir X-1 and star 2 at each wavelength. We then determined the wavelength calibration using the CuAr arc lamp exposures. We fit a low-order polynomial to the arc line wavelengths as a function of pixel number: the rms scatter of the fits was $\sim 1 / 4$ of a pixel. A rough flux-calibration was performed by comparing with the spectrum of the observed flux standard LTT 4364, though since the night was nonphotometric, this flux calibration should be considered only approximate.

\subsection{Infrared observations}

$K$-band spectroscopy of Cir X-1 was obtained using the Cryogenic Array Spectrometer/Imager (CASPIR) on the ANU $2.3 \mathrm{~m}$ telescope at Siding Spring Observatory on the night of 1997 June 20 . The $K$ grism was used with the SBRC $256 \times 256 \mathrm{InSb}$ array, giving a dispersion of $21^{\prime \prime} .5 \AA \mathrm{pixel}^{-1}$ over a wavelength range of $1.94-2.49 \mu \mathrm{m}$. The spatial scale was $0 .{ }^{\prime \prime} 5 \mathrm{pixel}^{-1}$. A $5^{\prime \prime}$ slit was used, oriented east-west: note that this means that Moneti's stars 2 and 3 both contributed light in our spectrum. The telescope was nodded by $\pm 12^{\prime \prime}$ along the slit to provide sky frames at the same position as the object. Argon lamp spectra were taken to perform wavelength calibration, and two nearby bright stars (BS 5699 and BS 5712) were observed in order to remove atmospheric spectral features and perform flux calibration.

Standard data reduction procedures were followed, using the local caspir package running in IRAF. Bias and dark frames were used to linearise all frames, the sky background was subtracted from the object frames, and pixel-to-pixel variations were corrected. The chip distortion was corrected in order to align the dispersion and spatial directions along rows and columns of the chip; the sky background was then subtracted and spectra extracted. A low-order polynomial was fit to the argon lines, and these calibrations applied to the object spectra. Flux calibration was achieved by dividing the observed spectra by the spectrum of a nearby mid Gtype star and then multiplying by a model for the absolute flux distribution of the calibrator. Residual terrestrial atmospheric features were then corrected using an early-type star.

\section{RESULTS}

\section{$3.1 \quad$ Optical spectra}

The optical spectrum is shown in Fig. 1. The spectrum clearly shows three narrow emission lines - $\mathrm{H} \alpha \lambda 6563$, He I $\lambda 6678$, and He I $\lambda 7065$ - as well as a broad component to the $\mathrm{H} \alpha$ line which is blue-shifted with respect to the narrow component. We fit gaussian models to these lines, using the specfit package in IRAF. After normalising the spectrum by 
Table 1. Fit to the optical spectrum. The spectrum was normalised by a low-order polynomial fit to the continuum, and four gaussians were fit to the lines, subject to the constraint that the velocity and width of the three narrow lines were the same. The table shows the resultant velocities, FWHMs, equivalent widths $W_{\lambda}$ of the gaussians, and the de-reddened line luminosities, assuming a reddening of $A_{V}=11$ (Predehl \& Schmitt 1995) and a distance of $5.5 \mathrm{kpc}$ (Case \& Bhattacharya 1998).

$\begin{array}{lcccc}\text { Component } & \begin{array}{c}\text { Velocity } \\ \left(\mathrm{km} \mathrm{s}^{-1}\right)\end{array} & \begin{array}{c}\text { FWHM } \\ \left(\mathrm{km} \mathrm{s}^{-1}\right)\end{array} & \begin{array}{c}W_{\lambda} \\ (\AA)\end{array} & \begin{array}{c}L_{\text {line }} \\ \left(\mathrm{erg} \mathrm{s}^{-1}\right)\end{array} \\ \text { H } \alpha \text { broad } & -310 \pm 40 & 2210 \pm 74 & 50 \pm 2 & 6.2 \times 10^{35} \\ \text { H } \alpha \text { narrow } & +378 \pm 12 & 402 \pm 8 & 74 \pm 1 & \\ \text { He I } \lambda 6678 & " & " & 3.4 \pm 0.5 & 1.5 \times 10^{34} \\ \text { He I } \lambda 7065 & " & " & 4.9 \pm 0.5 & 2.1 \times 10^{34}\end{array}$

a low-order polynomial fit to the continuum, we fit a single gaussian to each of the helium lines and two gaussians to the $\mathrm{H} \alpha$ line. The velocities and widths of the three narrow lines are consistent with being the same; we therefore constrained them to be equal. The details of the fit are shown in Table 1 . The narrow lines show a velocity of $\sim+378 \mathrm{~km} \mathrm{~s}^{-1}$ and a width of $\sim 9.5 \AA$, or $400 \mathrm{~km} \mathrm{~s}^{-1}$, while the broad component to the $\mathrm{H} \alpha$ line has a very different velocity $\left(-310 \mathrm{~km} \mathrm{~s}^{-1}\right.$, or $-688 \mathrm{~km} \mathrm{~s}^{-1}$ with respect to the narrow lines) and width (46 ̊, or $2200 \mathrm{~km} \mathrm{~s}^{-1}$ ).

No stellar features can be seen in the spectrum. In particular, no absorption features can be discerned which might sugggest the nature of the binary companion. There is no significant variability apparent between the five spectra.

Mignani, Caraveo \& Bignami (1997) obtained a spectrum of Cir X-1 using the HST Faint Object Spectrograph near periastron (phase 0.18) in June 1995. The line profile of the $\mathrm{H} \alpha$ line in their spectrum was very different to our 1997 spectrum. They found the broad component of the gaussian to be centered at approximately its rest wavelength, while the narrow component was observed at a velocity of $+380 \mathrm{~km} \mathrm{~s}^{-1}$. They interpreted the narrow component as arising in an accretion disc, with the $\sim 400 \mathrm{~km} \mathrm{~s}^{-1}$ velocity representing the rotation velocity of the disc material; the absence of the blue component they interpreted as a phasedependent shadowing effect. Under this model, the velocity of the narrow component should shift with orbital phase, while the broad component remains fixed.

Our spectrum was taken at apastron, and does not show the predicted shift of the narrow component. In fact, the velocities we observe for the narrow component of the $\mathrm{H} \alpha$ line (and the helium lines, not observed in the HST spectrum) are identical with the HST spectrum, while the broad component has shifted in velocity. This would seem to indicate that the model proposed by Mignani et al. for the system is wrong.

To investigate this, we used data from the AAT archive, which contains nearly all observations taken with the AAT since its inception. Cir X-1 has been observed using the AAT several times by different observers, with most observations occurring about 20 years ago. We obtained spectra at a total of five epochs which showed detectable emission: these are listed in Table 2 and shown in Fig. 2. The May 1976 data were published by wmw +77 (;) the rest of the data are unpublished. We obtained the original data from the AAT archive and reduced them using standard techniques and

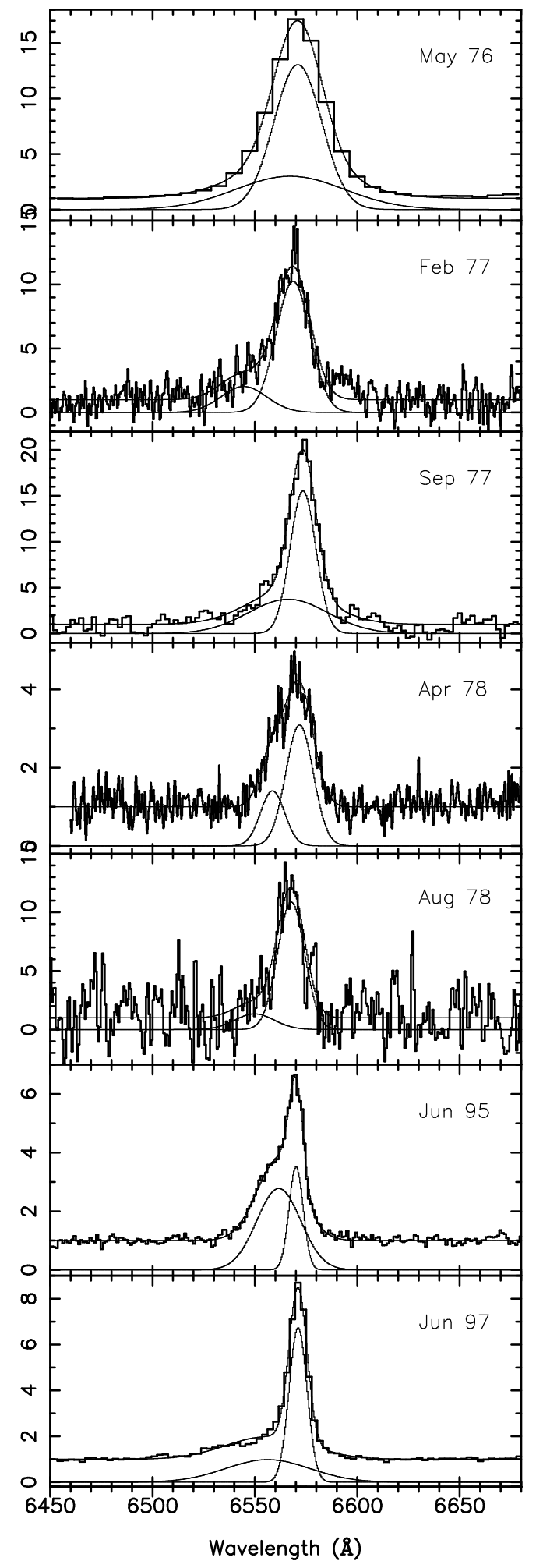

Figure 2. Line profiles of $\mathrm{H} \alpha$ in the archival observations of Cir $\mathrm{X}-1$, showing the two gaussians fit to each line and their sum. Each spectrum had been binned to twice the spectral resolution, and normalised by a polynomial fit to the continuum, so the $y$ axis shows the relative flux of the line. The last two spectra show the HST observation (June 95) and our new AAT observation (June 97). 
Table 2. List of observations of Cir X-1, including the archival AAT observations, the HST observation (June 1995), and our new AAT observation (June 1997). Column 2 gives the instrument/detector combination where 'B\&C' indicates the Boller \& Chivens spectrograph, 'RGO' indicates the RGO spectrograph with either the long $(82 \mathrm{~cm})$ or short $(25 \mathrm{~cm})$ camera; columns $3-5$ show the instrumental resolution, total exposure time, and orbital phase of the observation. The following columns show results of the fit to the H $\alpha$ line (see text for details), with gaussian 1 being the red-shifted (narrow) component, and gaussian 2 the blue-shifted (broad) component. The phase was calculated according the ephemeris of Stewart et al. (1991), and the fits are shown in Fig. 2.

\begin{tabular}{|c|c|c|c|c|c|c|c|c|c|c|}
\hline \multirow[b]{2}{*}{ UT Date } & \multirow[b]{2}{*}{ Instrument } & \multirow[b]{2}{*}{$\begin{array}{l}\Delta \lambda \\
(\AA)\end{array}$} & \multirow[b]{2}{*}{$\begin{array}{c}t_{\exp } \\
(\mathrm{s})\end{array}$} & \multirow[b]{2}{*}{ Phase } & \multirow{2}{*}{$\begin{array}{c}\text { velocity } \\
\left(\mathrm{km} \mathrm{s}^{-1}\right)\end{array}$} & & \multicolumn{3}{|c|}{ gaussian 2} \\
\hline & & & & & & $\begin{array}{l}\text { FWHM } \\
\left(\mathrm{km} \mathrm{s}^{-1}\right)\end{array}$ & $\begin{array}{l}W_{\lambda} \\
(\AA)\end{array}$ & $\begin{array}{c}\text { velocity } \\
\left(\mathrm{km} \mathrm{s}^{-1}\right)\end{array}$ & $\begin{array}{c}\text { FWHM } \\
\left(\mathrm{km} \mathrm{s}^{-1}\right)\end{array}$ & $\begin{array}{l}W_{\lambda} \\
(\AA)\end{array}$ \\
\hline 1976 May 21 & IDS & 15 & 3840 & 0.536 & $370 \pm 40$ & 1050 & 380 & & 340 & $200 \pm 30$ \\
\hline $1977 \mathrm{Feb} 5$ & RGO25/IPCS & 0.8 & 800 & 0.234 & 0 & $890 \pm 70$ & $214 \pm$ & -830 & $1200 \pm 160$ & $57 \pm 10$ \\
\hline 1977 Sep 4 & RGO25/IPCS & 4.3 & 2000 & 0.947 & $490 \pm$ & $640 \pm 50$ & $245 \pm 25$ & 160 & $2080 \pm 190$ & $180 \pm 25$ \\
\hline 1978 Apr $29-30$ & RGO82/IPCS & 0.45 & 10900 & 0.315 & $410 \pm 40$ & $720 \pm 55$ & $52 \pm 6$ & $-190 \pm 70$ & $630 \pm 80$ & $21 \pm 6$ \\
\hline 1978 Aug 11 & RGO25/IPCS & 1.3 & 2000 & 0.522 & $220 \pm 200$ & $700 \pm 600$ & $180 \pm 130$ & $-600 \pm 2000$ & $1030 \pm 120$ & $30 \pm 100$ \\
\hline 1995 Jun 1 & HST/FOS & 2.2 & 5160 & 0.183 & $330 \pm 10$ & $355 \pm 15$ & $30 \pm 2$ & $-50 \pm 20$ & $1145 \pm 25$ & $75 \pm 2$ \\
\hline 1997 Jun 4 & RGO82/TEK & 5.4 & 9000 & 0.510 & $380 \pm 12$ & $400 \pm 10$ & $74 \pm 2$ & $-300 \pm 50$ & $2210 \pm 100$ & $50 \pm 2$ \\
\hline
\end{tabular}

the FIGARO data reduction package. Note that for all these observations, light from stars 2 and 3 was presumably in the slit. Because of this, no attempt has been made to flux calibrate the spectra; instead, each spectrum was binned to a spectral sampling of two data points per resolution element, and the resulting spectrum was normalised by a low-order polynomial fit to the spectrum.

We performed the same double gaussian fit to these spectra as described in Table 14, and the results are shown in Table 2. Several points are immediately clear: despite the wide range of orbital phases, the broad component is never observed redward of the narrow component 1 . The (weighted) mean velocity of the narrow component is $+350 \mathrm{~km} \mathrm{~s}^{-1}$, that of the broad component $-100 \mathrm{~km} \mathrm{~s}^{-1}$. The scatter of the velocities of the broad component is much greater than that of the narrow component. No strong trends can be seen with either date of observation or orbital phase, with the exception of the equivalent width of the narrow component, which has been generally decreasing since 1976 (except for the extremely low value in April 1978).

\subsection{Infrared spectra}

Infrared spectra were obtained one orbit after the optical spectra. The mean spectrum is shown in Fig. 3. Bright emission lines of $\mathrm{Br} \gamma$ and He I $\lambda 2.058 \mu \mathrm{m}$ were visible in the spectrum. These two lines are generally observed in the K-band spectra of low- and high-mass X-ray binaries (bsc+97,bsct98,csfm99) and may arise in accretion discs or larger emitting regions, such as dense winds from the mass donors and/or ejecta from the binaries. There also seems to be a hint of asymmetry in the blue wing of the infrared lines, so we again fit gaussians to the line profile. We modelled each emission line as the sum of two gaussians, constraining the widths and velocities of the blue and red components to be the same for both lines. The results are shown in Table 3 .

Both lines are well fit by one gaussian at $+450 \mathrm{~km} \mathrm{~s}^{-1}$, a velocity very similar to the narrow red-shifted optical

$\dagger$ Except for the Apr 78 spectrum, where the width of the redshifted component is marginally smaller than the width of the blue-shifted component; however, the two widths are consistent with being identical.

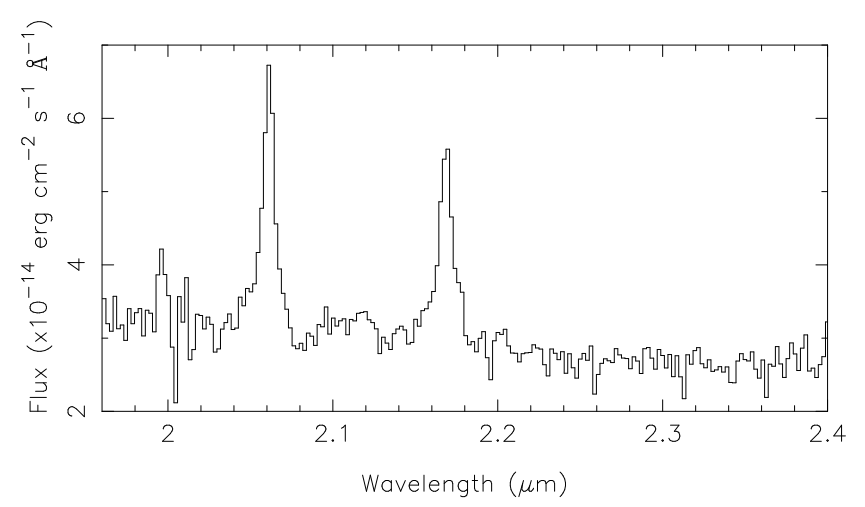

Figure 3. Infrared spectrum of Cir X-1, taken near apastron on 1997 June 20.

Table 3. Fit to the infrared spectrum. The spectrum was normalised by a low-order polynomial fit to the continuum, and four gaussians were fit to the lines, subject to the constraint that the velocity and widths of the blue (B) and red (R) components were the same for both species. The final column shows the dereddened integrated line luminosities, assuming a reddening of $A_{V}=11$ and a distance of $5.5 \mathrm{kpc}$.

$\begin{array}{lcccc}\text { Component } & \begin{array}{c}\text { Velocity } \\ \left(\mathrm{km} \mathrm{s}^{-1}\right)\end{array} & \begin{array}{c}\text { FWHM } \\ \left(\mathrm{km} \mathrm{s}^{-1}\right)\end{array} & \begin{array}{c}W_{\lambda} \\ (\mu \mathrm{m})\end{array} & \begin{array}{c}L_{\text {line }} \\ \left(\mathrm{erg} \mathrm{s}^{-1}\right)\end{array} \\ \text { H I Br } \gamma(\mathrm{R}) & 450 \pm 40 & 1170 \pm 90 & 88 \pm 7 & 4.3 \times 10^{34} \\ \text { He I (R) } & " & " & 96 \pm 7 & 3.1 \times 10^{34} \\ \text { H I Br } \gamma(B) & -1870 \pm 570 & 1020 \pm 440 & 12 \pm 6 & \\ \text { He I (B) } & " & " & 11 \pm 5 & \end{array}$

component, plus a blue-shifted component at a velocity of $\sim-2000 \mathrm{~km} \mathrm{~s}^{-1}$.

\section{AN ECCENTRIC LOW-MASS BINARY MODEL FOR CIR X-1}

Initially, it was suggested that Cir X-1 is a high mass binary consisting of a compact star (either a neutron star or black hole) and an OB supergiant companion star $(\mathrm{wmw}+77, \mathrm{mjh}+80)$. Its $\mathrm{X}$-ray properties imply a very eccentric binary orbit. The 16.6-day modulation in the X-ray luminosity is due to orbital variations in the mass accretion 


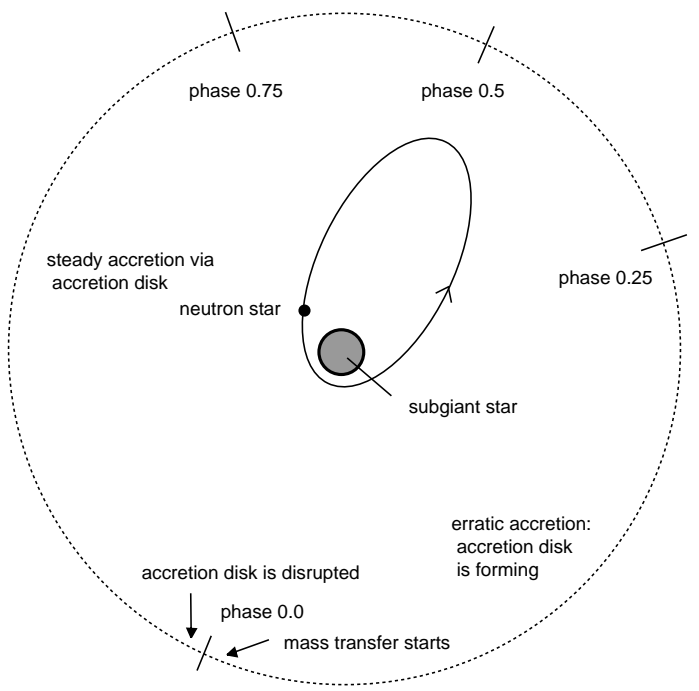

Figure 4. Model for Cir X-1. During periastron (phase 0) the neutron star passes its closest to the companion (mass $\sim 3$ $5 \mathrm{M}_{\odot}$ ), and the disc is disrupted, resulting in large X-ray fluctuations. After periastron passage, an accretion disc begins to form; mass accretion steadies, until after apastron (phase 0.5) steady accretion via a disc takes place. Tidal interactions begin to disrupt the disc again as periastron approaches.

of the compact star. The X-ray and radio bursts occur when the compact star encounters the dense stellar wind from the supergiant (ff80), or when tidal mass transfer is induced during the periastron passage (hay87 ). The Type I X-ray bursts that were discovered in a brief epoch in the mid-1980s (tfs86 ) indicated that the compact star is a neutron star. Recent observations (e.g. snp+91,gla94 ) indicate that the companion star is unlikely to be a massive supergiant star. No Type I bursts have yet been reported in RXTE observations.

Here, we propose a low-mass binary model, where the system consists of a neutron star orbiting around a subgiant companion star of about 3 to $5 \mathrm{M}_{\odot}$ (Fig. 旬; see also tw98 ). The orbital eccentricity is $\sim 0.7-0.9$. During the periastron passage, the companion star overfills its Roche-lobe, causing a transfer of mass at a super-Eddington rate onto the neutron star. As Cir X-1 is a X-ray burster, the magnetic field of the neutron star is relatively weak, and so the accretion flow is probably quasi-spherical during the periastron passage. Because of the large radiative pressure of emission from the neutron star, there must also be a strong (anisotropic) matter outflow. The inflow/outflow geometry may be related to the larger scale jets observed from the system, with symmetrical collimated outflows along some preferred axis.

After the periastron passage, the companion star is detached from its critical Roche-surface, and mass transfer ceases. Accretion continues as the neutron star captures the residual matter in its Roche-lobe. An accretion disc is gradually formed. The disc is geometrically thick, because the accretion rate is sufficiently high and is close to the Eddington limit. The accretion becomes more steady when disc accretion takes over from the less stable super-Eddington quasispherical accretion. Accretion via a disc continues to operate till the next periastron passage, where the accretion disc is disrupted by tidal interaction. When the periastron passage proceeds, the companion star overfills its Roche-lobe again, leading to another cycle of super-Eddington mass transfer, formation of the accretion disc, steady accretion via the disc and disruption of the accretion disc.

The periodic X-ray light curve of Cir X-1 has changed dramatically since its discovery in the 70 s (e.g. khbs76,tkm+89,sblm96 ). This indicates that the accretion mode has varied substantially in the last 25 years. Despite the fact that the $\mathrm{H} \alpha$ lines in the optical spectra obtained in the 70s and 90s show certain similarities, such as an asymmetric profile with a broad blue wing, it is inappropriate to assume that the optical spectra are the same at the same orbital phases in the $70 \mathrm{~s}$ and the $90 \mathrm{~s}$. The FWHM of the red (narrow) component of the $\mathrm{H} \alpha$ line seems to decrease from $\approx 700 \mathrm{kms}^{-1}$ in the $70 \mathrm{~s}$ to $\approx 380 \mathrm{~km} \mathrm{~s}^{-1}$ in the $90 \mathrm{~s}$. The central wavelength, however, does not show significant velocity variation, and its average value is $350 \pm 80 \mathrm{~km} \mathrm{~s}^{-1}$.

Our interpretation is that the red (narrow) component is emitted from irradiatively-heated matter which is relatively stationary with respect to the centre of mass of the system and has a low velocity dispersion. One of the natural choices is the heated surface of the companion star, on which the projected velocity of the matter is much less than the flow velocity near the neutron star.f

The velocity of the sharp red component of the $\mathrm{H}$ I Br $\gamma$ and the HeI $\lambda 2.058 \mu \mathrm{m}$ lines is $450 \pm 40 \mathrm{~km} \mathrm{~s}^{-1}$, which is consistent with that for the optical lines. Thus, the lines must also be emitted from a region relatively stationary to the centre-of-mass of the binary. The width of the IR lines, however, is much larger than the width of the optical lines. It is therefore unlikely that the emission site is the surface of the companion. One possibility is that these IR lines are emitted from a heated dust shell - the residue of ejecta from previous epochs.

The observations accumulated since the 70 s show that the broad component is blueward of the narrow component, except (possibly) in the 1978 April observation. The line widths were generally above $1000 \mathrm{~km} \mathrm{~s}^{-1}$, and it was even larger than $2000 \mathrm{kms}^{-1}$ in the 1976 and 1997 observations. Although the line centre velocities appear to show large variations (from $-600 \mathrm{~km} \mathrm{~s}^{-1}$ to $200 \mathrm{~km} \mathrm{~s}^{-1}$ ), they show no obvious correlation with orbital phase.

We suspect that the broad component is related to the high velocity flow from regions near the neutron star. X-ray observations have shown that the rate of mass accretion onto the neutron star is above the Eddington limit (e.g. ino89) . It is therefore possible that an outflow is driven by the Eddington luminosity. As we only see the blueward component, the lines are probably emitted from the optically thick

$\ddagger$ Note that, if the narrow emission is arising from the heated face of the secondary, the equivalent width should vary in a smooth fashion through the binary orbit, with a maximum at phase 0.5; such a pattern is not seen in the data in Table 2. However, we argue that the mass transfer rate has varied so much over the 21 y spanned by the observations that it is misleading to try to compare the equivalent widths. Indeed, if we consider three groups of spectra near to each other in time (1976-1977, 1978, and 1995-1997) there is a (slight) tendency for the equivalent width to be highest at phase 0.5 . Such a relation certainly needs to be confirmed. 


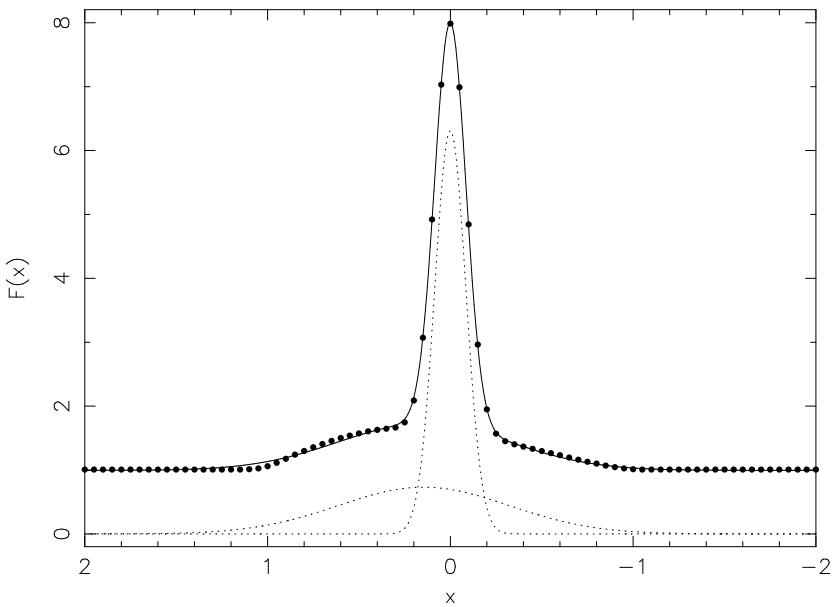

Figure 5. Line profile of emission from an expanding envelope, with parameters as described in the text. Positive $\mathrm{x}$ corresponds to the blue-shifted frequency; negative $\mathrm{x}$ corresponds to the redshifted frequency. The filled circles are the simulated data. The solid line is a fitted model with a constant and two gaussians. The two gaussians are represented by the dotted lines. The ratio of the widths of the broad and narrow gaussians is 5.5, and the ratio of the width of the broad gaussian to the separation of the two gaussian peaks is 3.22 .

outflowing matter, of which the red-shifted components are heavily absorbed or obscured. The changes in the line centre velocities and widths deduced from the fits are probably the result of the changes in optical depth over the different observational epochs.

The quality of our data precludes us performing a fit of this model to the line profile; rather, we present it as a possible explanation for the observed asymmetric line profile. We have constructed a simple toy model to demonstrate the asymmetric line profile that might be observed from such an outflow. In our model the broad line emission region is a spherically symmetric extended envelope, with outer and inner radii $R_{\text {out }}$ and $R_{\text {in }}$ respectively. The envelope encloses an opaque sphere with radius equal to $R_{\text {in }}$. We have considered various functional forms for the velocity, emissivity and absorption coefficient distribution, and have found that models with a power-law velocity profile generally can produce a broad line profile similar to the observations (see Appendix).

In Fig. 5, we show a simulated a blue-shifted broad line from an expanding envelope with outer and inner radii, $R_{\text {out }}=1$ and $R_{\text {in }}=0.3$ respectively. The radial expanding speed $V$ has a power-law profile with an index of +1 , and it is normalised such that its value is 1 at $R_{\text {out }}$. The normalised local Doppler width of the line is 0.1. The emissivity is uniform within the emitting region, with $\eta_{c}=\eta_{l}=1$. The continuum and line absorption coefficients are uniform, and their ratio $\chi_{l} / \chi_{c}=0.35$. The total effective absorption optical depth $\tau$ is such that $\left(\chi_{c}+\chi_{l}\right) R_{\text {out }}=1$.

As the narrow line is emitted from the heated companion star, we simply assume it is a gaussian with a width of 0.123 and a norm of 4.0. This will give an approximate ratio of $2 / 3$ for the equivalent widths of the simulated broad and narrow line components.

As shown, the resultant line profiles resembles the $\mathrm{H} \alpha$ line observed in our June 1997 data. If we fit the simulated line with two gaussians and a constant, the relative widths and shifts of the two gaussian components are in good agreement with the fitted parameters of the June 1997 data (Fig. 5).

The blue components of the $\mathrm{HI} \mathrm{Br} \gamma$ and the HeI $\lambda 2.058 \mu \mathrm{m}$ lines have a velocity shift of $-1870 \pm 570 \mathrm{~km} \mathrm{~s}^{-1}$ and a width of $1020 \pm 440 \mathrm{~km} \mathrm{~s}^{-1}$. If we interpret the blue component of the IR lines as being emitted from the vicinity of the neutron star, the difference in the velocities between the optical and IR lines is due to optical depth effects and/or the uncertainties in determining the central velocities of the lines with the spectral resolution of the IR spectrum.

mjh+80 (a) ssumed a $1 \mathrm{M}_{\odot}$ compact star, and by comparing the radius of various classes of stars with the separation of the two component star at periastron, they estimated the mass of the companion star and the orbital eccentricity for which tidally induced mass transfer can occur during the periastron passage. In our model, the mass transfer is driven by the overflow of the mass-donor star's Roche-lobe instead of the stellar wind from the star, and it therefore requires the companion star to fill its Roche-lobe at periastron, i.e. the star's radius $R_{2}$ to be equal to or larger than its 'instantaneous' critical Roche-lobe surface. It is beyond the scope of this paper to derive an exact phase-dependent effective Roche-lobe surface. However, as a first approximation, if we simply take the expression of the Roche-lobe radius for circular systems (see kop59) and replace the orbital separation term by the separation between the two stars of Cir X-1 at periastron, then we can obtain an effective Roche-lobe radius at periastron (phase 0 ):

$R_{\mathrm{L}} \approx a(1-e)[0.38+0.2 \log q]$

where the mass ratio $q=M_{2} / M_{1}$ and $a$ is the semi-major axis. Using this approximate expression for $R_{\mathrm{L}}$, we can constrain the appropriate parameter space $\left(e ; M_{1}, M_{2}\right)$ where Roche-lobe overflow occurs during the periastron passage for various types of mass-donor stars.

In Fig. 6] we show the mass-radius relation for supergiant, giant and main-sequence stars, together with $R_{\mathrm{L}}$ for eccentric systems with a 16.6 day period and a $1.4 \mathrm{M}_{\odot}$ neutron star primary. If the companion star is a main sequence star, its mass is about $10 \mathrm{M}_{\odot}$ and the orbital eccentricity $e>0.9$ in order to fill its Roche-lobe at periastron. A subgiant star with $M_{2} \approx 3-5 \mathrm{M}_{\odot}$ can easily fill its Roche-lobe for an orbital eccentricity of about 0.7-0.9.

The argument for a low mass system is generally consistent with the combined results of our spectroscopic observations and the study on the effects of supernova kicks on neutron star binaries. A simulation study (bp95b) showed that the eccentricity of HMXBs (with $M_{2} \sim 10 \mathrm{M}_{\odot}$ ) and LMXBs (with $M_{2} \sim 1-5 \mathrm{M}_{\odot}$ ) resulting from supernova explosions with isotropic kicks will be about $0.5(\sigma \approx 0.2)$ and $0.8(\sigma \approx 0.05)$ respectively if the final orbital period is about 16 days. The corresponding centre-of-mass velocities are $50(\sigma \approx 12)$ and $150(\sigma \approx 100) \mathrm{kms}^{-1}$. If we accept the interpretation that the narrow line components originate from the heated companion star, the line-of-sight component of the centre-of-mass velocity of the system is about $350 \mathrm{~km} \mathrm{~s}^{-1}$, or $430 \mathrm{~km} \mathrm{~s}^{-1}$ after applying a correction for Galactic rotation (using the rotation curve of cle85 ) . This correction was made assuming that the position of 


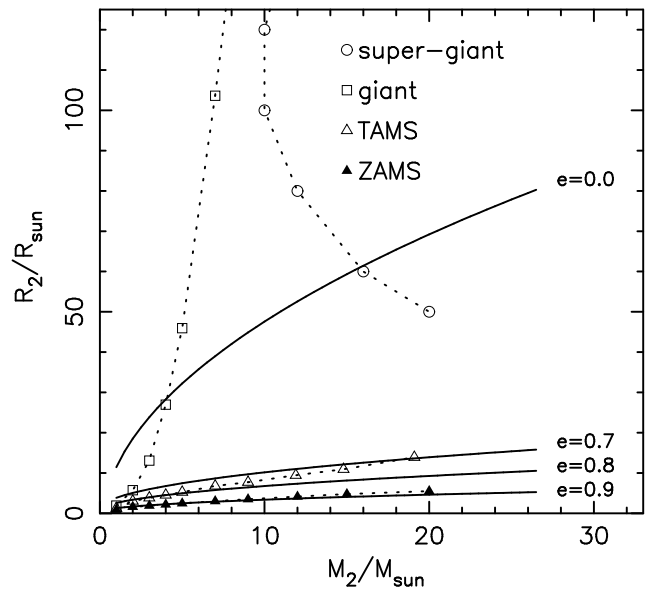

Figure 6. The radius of the Roche-lobe at periastron as a function of the mass of the companion star, calculated from an eccentric system with a $16.6 \mathrm{~d}$ period and a $1.4 \mathrm{M}_{\odot}$ neutron star primary. For Roche-lobe overflow to occur, the size of the secondary must exceed or equal the volume enclosed by the instantaneous critical Roche-lobe surface. We also show the size of the star for various masses at the zero-age main-sequence (ZAMS), terminated main-sequence (TAMS), giant and supergiant stages (indicated with filled triangles, open triangles, open squares and open circle respectively). The radii of the stars are derived from the evolutionary models of Maeder \& Meynet (1988), with the ZAMS, TAMS and giant stages corresponding to Point 1, 7 and 14 in the evolutionary tracks. The mass-radius relation of the super-giant stars, which is not as well-defined, is adopted from Lang (1991).

G321.9-0.3 was the birthplace of Cir X-1, but this makes little difference to the resulting correction. By combining with the transverse velocity $\left(\sim 300-400 \mathrm{~km} \mathrm{~s}^{-1}\right)$ inferred from the age and the distance of the supernova remnant G321.9-0.3 (cpc75 ) we obtain an estimated centre-of-mass velocity of $\sim 540 \mathrm{~km} \mathrm{~s}^{-1}$. This velocity could be substantially higher, as it depends on the (very uncertain) age estimate of the supernova remnant; transverse velocities as high as $1600 \mathrm{~km} \mathrm{~s}^{-1}$ are possible (schn93 ). A velocity of $\sim 540 \mathrm{~km} \mathrm{~s}^{-1}$ is about $3 \sigma$ from the mean centre-of-mass velocities obtained from the simulation study by bp95b (.) Such high velocities are not at all consistent with the simulations for high mass systems: even the low velocity is about $13 \sigma$ above the mean velocity predicted for a high-mass system.

The implied velocity for Cir X-1 makes it one of the fastest moving binaries known: radial velocities of $\sim$ $300 \mathrm{~km} \mathrm{~s}^{-1}$ have been measured for a handful of other LMXBs, e.g. 4U 1556-605 (mpmb89 ), GX 349+02 (pa91) , 4U 1957+11 (mtb78) ; see joh92 (f) or a discussion. Such velocities strengthen the identification of Cir X-1 as a lowmass system, as the fastest moving HMXBs have velocities $v<100 \mathrm{~km} \mathrm{~s}^{-1}$. If the transverse velocity is similar to the

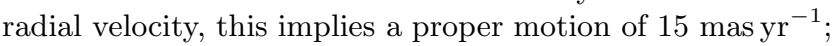
in an ongoing project to measure the proper motion of the radio counterpart to $\mathrm{Cir} \mathrm{X}-1$, we can measure a relative positional accuracy of around 40 mas, so we should measure the proper motion in a couple of years.

An alternative simulation by shi98 (a) lso concluded that Cir X-1 is a low-mass system. However, his simulation requires the system to be old $\left(\sim 10^{7} \mathrm{y}\right)$, which seems difficult to reconcile with the observations that Cir X-1 is associated with the supernova remnant G321.9-0.3.

The RXTE observations show that the current X-ray luminosity is quite steady in the orbital phases around 0.5-1.0. The cycle-to-cycle variations are insignificant in comparison with the those at the earlier orbital phases. We interpret the steady accretion as due to the presence of an accretion disc. The erratic cycle-to-cycle luminosity variations are the consequence of the absence of the disc acting as a buffer; cf. the rms fluctuations in the RXTE data at phases 0.15 and 0.5 (sblm96 ) . Moreover, we do not see double-horn features in the $\mathrm{H} \alpha$ line, such as those often observed in cataclysmic variables (e.g. hm86 ) or black hole binaries (e.g. swhw98) . This, together with the near-Eddington luminosity of the $\mathrm{X}$-rays during the disc-accretion phase, implies that the disc is non-Keplerian and it is both geometrically and optically thick. As the matter is not tightly bound by the gravity of the neutron star, the large variation in the tidal force when the neutron star approaches periastron could easily disrupt the disc, causing a short temporal decrease in the accretion luminosity of the system.

What constraints can we put on the age of the system? In our model, mass transfer at periastron does not begin until the star has evolved from the main-sequence towards the giant branch. For a $3-5 \mathrm{M}_{\odot}$ star, the subgiant stage may last $\sim 5 \times 10^{5} \mathrm{y}$. If Cir X-1 is associated with the nearby supernova remnant G321-0.3, then the implied age of the system as an eccentric neutron star binary is $<10^{5} \mathrm{y}$. The Eddington limit for the rate of mass transfer onto a neutron star is $\sim 3 \times 10^{8} \mathrm{M}_{\odot} \mathrm{y}^{-1}$, giving a mass transfer time scale of $3 \times 10^{7}$ y. Since the companion star in Cir X-1 can lose mass at a rate greater than this as an average in each orbital cycle, the life-span of the system as an X-ray binary is correspondingly shorter, but still comfortably above either the evolution time scale of the companion star or the age of the supernova remnant.

\section{CONCLUSIONS}

We have detected strong optical and infrared emission lines in the spectrum of Cir X-1; the $\mathrm{H} \alpha$ and infrared lines are asymmetric, with a narrow component at a velocity of $\sim+350 \mathrm{~km} \mathrm{~s}^{-1}$ and a broader, blue-shifted component. Archival optical observations show that an asymmetric $\mathrm{H} \alpha$ emission line has been in evidence for the past twenty years, although the shape of the line has changed significantly. The narrow component is always seen redwards of the broad component, at a velocity around $200-400 \mathrm{~km} \mathrm{~s}^{-1}$; the broad component has a much larger scatter of velocities. These properties are not consistent with the interpretation of mcb97 (,) who suggested that the narrow component arises in an accretion disc, with the absence of a red component due to a phase-dependent shadowing effect.

Instead, we suggest that the narrow component arises from the heated surface of the companion star, which is a subgiant of about $3-5 \mathrm{M}_{\odot}$, while the broad component arises in an optically thick, high velocity outflow driven by super-Eddington accretion onto the neutron star. During periastron passage, the companion star overfills its Rochelobe, causing a transfer of mass at a super-Eddington rate, 
which in turn drives a strong matter outflow. After periastron, mass transfer from the companion ceases, but accretion continues at a near-Eddington rate as the neutron star captures the residual matter in its Roche-lobe. An accretion disc gradually forms, which is non-Keplerian and geometrically thick. This change between quasi-spherical and disc accretion causes the change between strong X-ray variability near phase 0 and steady accretion between phases 0.5-1.0. Thus our observations are in general consistent with a lowmass binary model for the system. In this model, the velocity of the narrow component reflects the space velocity of the binary; the implied radial velocity $\left(+430 \mathrm{~km} \mathrm{~s}^{-1}\right)$ makes Cir X-1 one of the fastest moving binaries known.

\section{ACKNOWLEDGMENTS}

We thank Allyn Tennant, Peter Wood, Lex Kaper, and Thomas Tauris for useful discussions; we also thank Peter for assistance with our IR observation. RPF was supported during the period of this research initially by ASTRON grant 781-76-017, and subsequently by EC Marie Curie Fellowship ERBFMBICT 972436. KW is supported by ARC through an Australian Research Fellowship. This research was based in part on observations made with the NASA/ESA Hubble Space Telescope, obtained from the data archive at the Space Telescope Science Institute. STScI is operated by the Association of Universities for Research in Astronomy, Inc. under NASA contract NAS 5-26555.

\section{APPENDIX A: LINE PROFILE SIMULATIONS}

The transfer equation that determines the intensity of the radiation is

$\left[\frac{1}{c} \frac{\partial}{\partial t}+(\hat{n} \cdot \nabla)+\chi(\nu, \vec{r})\right] I(\nu, \vec{r})=\eta(\nu, \vec{r})$,

where $I(\nu, \vec{r})$ is the intensity, $\hat{n}$ is the unit vector in the direction of the ray propagation, $\eta(\nu, \vec{r})$ is the emissivity and $\chi(\nu, \vec{r})$ is the extinction coefficient. We assume a (quasi)stationary state, such that the term $\partial / \partial t$ can be set to zero. In a general situation, the extinction coefficient consists of an absorption term and a scattering term. Therefore, one needs to solve an integro-differential equation to determine the radiation field and then the emerging intensity. However, if we can neglect scatterings, the situation is greatly simplified and a simple formal solution can be obtained.

In our model, we assume that the radiation is emitted from an envelope within which matter is moving with velocity $\vec{v}(\vec{r})$. Because the emitters are in relative motion to the observer, there are velocity shifts in the frequencies of the radiation they emit. There is also a velocity spread among the emitters, and so the frequency-dependence of the local line absorption and emission coefficients are modified.

For mathematical convenience we consider a dimensionless frequency $x\left(\equiv c\left(\nu-\nu_{o}\right) / \nu_{o} v_{*}\right)$, and a dimensionless velocity $\vec{V}(\vec{r})\left(\equiv \vec{v}(\vec{r}) / v_{*}\right)$, where $\nu_{o}$ is the frequency in the rest frame, $v_{*}$ is a velocity normalisation and $c$ is the speed of light. The emissivity and the absorption coefficients are decomposed into a continuum and a line component. As a

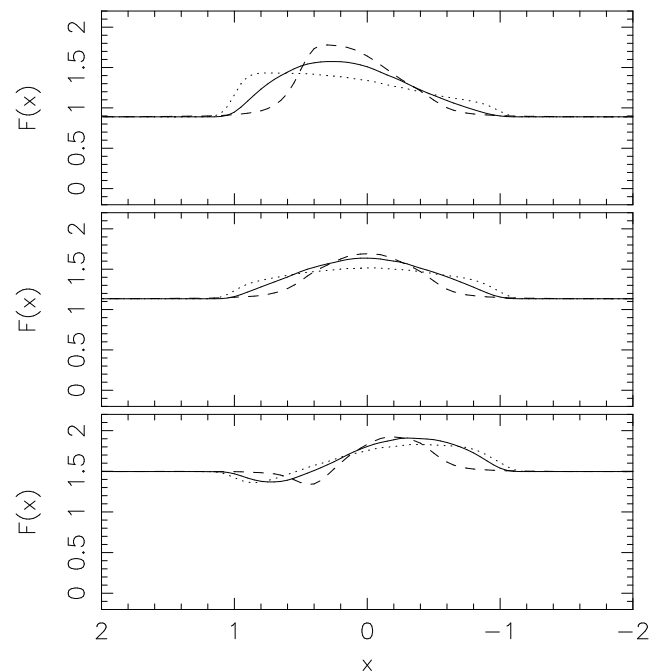

Figure A1. Simulated broad line components from expanding envelopes for a power-law radial velocity profile with various power-law indices. From top to bottom, $\chi_{l} / \chi_{c}=0.1,1$ and 10 . The other parameters are: $\eta_{l}=1, \eta_{c}=1, R_{\text {out }}=1, R_{\text {in }}=0.1$, $\delta=0.1$ and $\left(\chi_{l}+\chi_{c}\right) R_{\text {out }}=1$. The velocity normalisations corresponding to the three cases with the power-law indices +1 (solid line), 0 (dotted line) and -1 (dashed line) are $V\left(R_{\text {out }}\right)=1,1$ and 0.45 respectively.

first approximation, we assume that the continuum component does not vary significantly with frequency near the line frequencies, and hence we have

$$
\begin{aligned}
& \eta(x, \vec{r})=\eta_{c}(\vec{r})+\eta_{l}(\vec{r}) \phi(x, \vec{r}, \vec{V}) ; \\
& \chi(x, \vec{r})=\chi_{c}(\vec{r})+\chi_{l}(\vec{r}) \phi(x, \vec{r}, \vec{V}) .
\end{aligned}
$$

In the above expression, $\phi(x, \vec{r}, \vec{V})$ is the line profile, given by

$\phi(x, \vec{r}, \vec{V})=\frac{1}{\delta(\vec{r}) \sqrt{\pi}} \exp \left\{-[x-(\hat{n} \cdot \vec{V})]^{2} / \delta^{2}(\vec{r})\right\}$,

where $\delta(\vec{r})$ is the normalised local Doppler width.

As the rays that reach a distance observer are parallel, we can assign each of them an impact parameter $p$ with respect to the centre of the emitting envelope. The flux at a frequency $x$ seen by the observer is the sum of all rays, i.e.

$F(x)=\int d \varphi \int d p p I^{*}(x, p)$

where $I^{*}(x, p)$ is the intensity of the ray emerging from nonobscured part of the emitting envelope $\Re$, and $\int d \varphi \int d p p$ is the projection area of the expanding envelope on the sky plane. If we neglect scatterings, $I^{*}(x, p)$ is the formal solution to the radiative transfer equation, and it is

$$
\begin{aligned}
I^{*}(x, p) & =I_{o}(x, p) \exp \left\{-\tau\left(x, p, z_{\min }(p)\right)\right\} \\
& +\int_{z_{\min }(p)}^{z_{\max }(p)} d z\left[\eta_{c}+\eta_{l} \phi\right] \exp \{-\tau(x, p, z(p))\}
\end{aligned}
$$

(e.g. mih78,kyf88 ), where $d z$ is a path element along the ray propagation, and $I_{o}(x, p)$ is the intensity of the background radiation. The optical depth $\tau(x, p, z(p))$ is

$\tau(x, p, z(p))=\int_{z(p)}^{z_{\max }(p)} d z\left[\chi_{c}+\chi_{l} \phi\right]$. 
If there is no strong background radiation behind the emitting envelope (i.e. $I_{o}(x, p) \approx 0$ ), then the observed flux is simply

$$
\begin{aligned}
F(x)=\int d \varphi \int d p p \int_{z_{\min }(p)}^{z_{\max }(p)} d z\left[\eta_{c}+\eta_{l} \phi\right] \\
\times \exp \{-\tau(x, p, z(p))\} .
\end{aligned}
$$

We consider a hybrid method to calculate the line flux $F(x)$. The optical depth $\tau(x, p, z(p))$ is determined by a direction integration of the absorption coefficient along the ray propagation $\hat{n} z$ (for fixed $p$ ). Instead of carrying out a direct integration in the 3 -D space $(\varphi, p, z)$, we use a Monte-Carlo method to determine $F(x)$.

In the Monte-Carlo calculation, we consider each emitter as a discrete source which emits a packet of line and continuum radiation spanning a certain frequency range. The packet is absorbed along the path of propagation subject to the optical depth. The strength of the emission from each emitter (i.e. $\eta_{c}$ and $\eta_{l}$ ) is weighted according to a distribution profile in the envelope. We consider 50000 emitters in each simulation. The observed flux is the sum of the contribution of all emitters in the non-obscured region $\Re$.

This quick-fix method allows us to handle complicated geometry due to obscuration easily. It also enables us to survey various functional forms for the distribution profiles of the velocity, emissivity and absorption coefficients and obtain qualitative features of the line emission with sensible computational time.

In Fig. A1 we show examples of line profiles for three cases in which the radial velocity distribution is a power-law function (with indices $-1,0$ and +1 respectively). In these cases, if the line absorption is weaker than the continuum absorption, i.e. $\chi_{l}<\chi_{c}$, an asymmetric blue-shifted profile can be obtained. If $\chi_{l} \approx \chi_{c}$, the line is symmetric with no obvious shift of the line centre frequency. If $\chi_{l}>\chi_{c}$, a PCygni profile will be obtained.

\section{REFERENCES}

Bandyopadhyay, R., Shahbaz, T., Charles, P. A., Naylor, T. 1998, Infrared spectroscopy of low-mass X-ray binaries II, MNRAS, submitted

Bandyopadhyay, R., Shahbaz, T., Charles, P. A., van Kerkwijk, M. H., Naylor, T. 1997, MNRAS, 285, 718

Brandt, N., Podsiadlowski, P. 1995, MNRAS, 274, 461

Case, G. L., Bhattacharya, D. 1998, ApJ, 504, 761

Clark, D. H., Parkinson, J. H., Caswell, J. L. 1975, Nat, 254, 674

Clark, J. S., Steele, I. A., Fender, R. P., Coe, M. J. 1999, Near IR spectroscopy of high mass X-ray binaries, A\&A, submitted

Clemens, D. P. 1985, ApJ, 295, 422

Croke, B. F. W. 1995, PASP, 107, 1255

Duncan, A. R., Stewart, R. T., Haynes, R. F. 1993, MNRAS, 265, 157

Fender, R., Spencer, R., Tzioumis, T., Wu, K., van der Klis, M., van Paradijs, J., Johnston, H. 1998, ApJ, 506, L121

Fransson, C., Fabian, A. C. 1980, A\&A, 87, 102

Glass, I. S. 1994, MNRAS, 268, 742

Goss, W. M., Mebold, U. 1977, MNRAS, 181, 255

Haynes, R. F. 1987, Aust. J. Phys., 40, 741

Haynes, R. F., Jauncey, D. L., Murdin, P. G., Goss, W. M., Longmore, A. J., Simons, L. W. J., Milne, D. K., Skellern, D. J. 1978, MNRAS, 185, 661
Haynes, R. F., Komesaroff, M. M., Little, A. G., Jauncey, D. L., Caswell, J. L., Milne, D. K., Kesteven, M. J., Wellington, K. J., Preston, R. A. 1986, Nat, 324, 233

Horne, K., Marsh, T. R. 1986, MNRAS, 218, 761

Inoue, H. 1989, in J. Hunt, B. Battrick (eds.), Proc. 23rd ESLAB Symp. on Two Topics in X-ray Astronomy, Bologna, Italy 13-20 September 1989, ESA, Paris, p. 109

Johnston, H. M. 1992, Ph.D. thesis, California Institute of Technology

Kaluzienski, L. J., Holt, S. S., Boldt, E. A., Serlemitsos, P. J. 1976, ApJ, 208, L71

Kopal, Z. 1959, Close Binary Systems, Vol. 5 of International Astrophysics Series, Chapman \& Hall, London

Kudritzki, R. P., Yorke, H. W., Frisch, H. 1988, Radiation in moving gaseous media: Eighteenth Advanced Course of the Swiss Society of Astrophysics and Astronomy, Geneva Observatory, Sauverny-Versoix

Lang, K. 1991, Astrophysical Data: Planets and Stars, SpringerVerlag, New York

Maeder, A., Meynet, G. 1988, A\&AS, 76, 411

Margon, B., Thorstensen, J. R., Bowyer, S. 1978, ApJ, 221, 907

Mignani, R., Caraveo, P. A., Bignami, G. F. 1997, A\&A, 323, 797

Mihalas, D. 1978, Stellar Atmospheres, Freeman, San Francisco, 2nd edition

Moneti, A. 1992, A\&A, 260, L7

Motch, C., Pakull, M. W., Mouchet, M., Beuermann, K. 1989, A\&A, 219, 158

Murdin, P., Jauncey, D. L., Haynes, R. F., Lerche, I., Nicolson, G. D., Holt, S. S., Kaluzienski, L. J. 1980, A\&A, 87, 292

Penninx, W., Augusteijn, T. 1991, A\&A, 246, L81

Predehl, P., Schmitt, J. H. M. M. 1995, A\&A, 293, 889

Shirey, R. E., Bradt, H. V., Levine, A. M., Morgan, E. H. 1996, ApJ, 469, L21

Shirey, R. W. 1998, Ph.D. thesis, MIT

Soria, R., Wickramasinghe, D. T., Hunstead, R. W., Wu, K. 1998, ApJ, 495, L95

Stewart, R. T., Caswell, J. L., Haynes, R. F., Nelson, G. J. 1993 , MNRAS, 261, 593

Stewart, R. T., Nelson, G. J., Penninx, W., Kitamoto, S., Miyamoto, S., Nicolson, G. D. 1991, MNRAS, 253, 212

Tennant, A. F. 1988, Adv. Space Res., 8(2-3), 397

Tennant, A. F., Fabian, A. C., Shafer, R. A. 1986a, MNRAS, 219, 871

Tennant, A. F., Fabian, A. C., Shafer, R. A. 1986b, MNRAS, 221, $27 \mathrm{p}$

Tennant, A. F., Wu, K. 1998, 25 year X-ray observations of Cir X1 , in preparation

Tsunemi, H., Kitamoto, S., Manabe, M., Miyamoto, S., Yamashita, K., Nakagawa, M. 1989, Publ. Astr. Soc. Japan, 41, 391

Whelan, J. A. J., Mayo, S. K., Wickramasinghe, D. T., Murdin, P. G., Peterson, B. A., Hawarden, T. G., Longmore, A. J., Haynes, R. F., Goss, W. M., Simons, L. W., Caswell, J. L., Little, A. G., McAdam, W. B. 1977, MNRAS, 181, 259 